\title{
Outlines of a Hybrid Model of the Process Plant Operator
}

\author{
Rasmussen, Jens
}

Published in:

Monitoring Behaviour and Supervisory Control

Publication date:

1976

Document Version

Publisher's PDF, also known as Version of record

Link back to DTU Orbit

Citation (APA):

Rasmussen, J. (1976). Outlines of a Hybrid Model of the Process Plant Operator. In T. B. Sheridan, \& G. Johannsen (Eds.), Monitoring Behaviour and Supervisory Control (pp. 371-384). Plenum Publishing Corporation.

\section{General rights}

Copyright and moral rights for the publications made accessible in the public portal are retained by the authors and/or other copyright owners and it is a condition of accessing publications that users recognise and abide by the legal requirements associated with these rights.

- Users may download and print one copy of any publication from the public portal for the purpose of private study or research.

- You may not further distribute the material or use it for any profit-making activity or commercial gain

- You may freely distribute the URL identifying the publication in the public portal

If you believe that this document breaches copyright please contact us providing details, and we will remove access to the work immediately and investigate your claim. 


\title{
OUtlines Of a HybRId MOdel OF THE PROCEss Plant OPERATOR ${ }^{1}$
}

\author{
Jens Rasmussen \\ Danish Atomic Energy Commission, \\ Research Establishment, Risø
}

\section{INTRODUCTION}

Modeling the performance of human operators as an integral part of a system is becoming a vital problem to process plant designers. Reviews of major system failures and accidents repeatedly find that the human element plays a major role in $70 \%$ of the cases (Cornell, 1968. Rasmussen, 1959. Scott, 1971). The increasing re liability and safety requirements caused by the rapid growth in production unit size force the designer to include consideration of abnormal plant conditions due to failures of extremely low probability. For such tasks the operator will not be able to compensate design deficiencies by his great adaptability, and the interface design can not evolve through trial and error. This situation is further accentuated by the rapid development of data processing and display equipment with potential for very complex man-machine interaction.

\section{THE PROBLEM}

In system analysis and design of modern process plants, the operator is generally considered a vital part of the control system. He is a flexible - and inventive - data processor transforming in formation-displayed by the plant into appropriate actions according to his current goal. His data processes must be controlled by a representation of some kind of the functional properties of the plant. This representation obviously can be derived from different sources; e.g. from prior experience with plant behavior; from knowledge of internal anatomy and functioning of the plant; or from prescribed rules and instructions. Furthermore, he may use different mechanisms for the processing; e.g. he may respond "automatically" to a situation or he may identify a problem and "think".

Such features of his data processing must change, when tasks related to infrequent, abnormal plant conditions replace familiar tasks. Reviews of incidents and accidents often reveal difficulties in the operator's proper identi-

1In: Monitoring Behavior and Supervisory Control (1976) Edited by Thomas B. Sheridan and Gunnar Johannsen. Plenum 
fication of system state and appropriate goal in unfamiliar situations. The cause can be the complexity and dynamic properties of the plant leading to tasks exceeding the ultimate data handling capacity of the operator. However, for process plants the problem typically is not whether he is able to per form the task, but rather whether he will do it under the actual work conditions; i.e. whether the appropriate mode of data processing is activated.

\section{Study Of Human PeRformance In Real Life Tasks}

Fixation of mental procedures which have proved efficient in frequent applications is a well known human trait. The difficulty of proper identification of serious abnormal plant conditions and the use of improper procedures often indicate such adherence to mental procedures which have proved to be efficient during normal task condition or during more frequent fault conditions - e.g. instrument failures. Therefore, a model of operator behavior related to plant reliability and safety can not be obtained by adding together results from isolated psychological experiments. It must be based upon study of the performance during the actual, real-life work condition.

The need to identify the internal mental processes of the operator without experimental perturbations of his task raises difficult methodological problems. In spite of the well known difficulties of the method, we have chosen verbal protocols as the source of information for the modeling effort. We have found it possible to get important information in this way, but the appropriate method of analysis and the difficulties met depend upon the type of task considered. Protocols obtained from trained process operators are difficult to analyze and the model we are seeking is needed to structure the analysis. An iterative method must be used for protocol analysis as well as verification by other experimental methods.

\section{VERBAL PROTOCOLS}

A significant part of the problem thus is to develop experimental techniques and a work rationale, so that meaningful results can be obtained from protocols. A number of protocols collected during start up of thermal power stations have been analyzed tentatively and some general observations will illustrate the problems:

-The process operator has several different tasks going on simultaneously, and each task has several steps or sub tasks implying basically different data processes at different abstraction levels.

-The protocols generally give very little information on the underlying data processes. They are rather a sequence of statements indicating the operator's "state of knowledge" regarding the operational state of the plant, 
operator tasks and actions, etc. Only when unfamiliar sub tasks turn up are more detailed processing of data and observations mentioned.

-Very few observations are formulated as quantitative reading of instruments, and then, typically, only if observations should be related to some-prescribed reference value. Observations are generally expressed at a higher level of abstraction related to normal plant state, expected state, next task etc. Rather than reading instruments, the operator seems to ask higher level questions to the system, and individual instrument readings are used as symbols for system states.

-Very little planning is mentioned regarding the tasks to be performed, the instruments to be read etc. The operator seems spontaneously to "know" what is going on and where to focus his attention.

These features of course could be due to difficulties in having operators verbalizing for longer periods, but it seems more reason able to assume the use of data processes which can not be verbalized. During a long period of interaction with a system a trained operator has developed a large repertoire of complex and partly subconscious routines, which are controlled by a conscious and "verbalizeable" sequence at a high level of abstraction.

This process appears not to be controlled by decisions considering alternatives, but by general conditioning by his "process feel", i.e. a subconscious dynamic plant model.

Processes at the level of observed information arc only ex pressed in unfamiliar tasks, e.g. during plant malfunctions. It is difficult to obtain a reasonable quantity of such sequences from plant operation, and as an example of this type of task we have used electronic maintenance technician's diagnostic task.

The protocols obtained from this task contain detailed information on the data processes used and 40 protocols have been analyzed in detail (Rasmussen and Jensen, 1974). The processes can be de scribed by a sequence of standard subroutines linked by heuristic rules. A few general features are relevant in the present context:

The basic nature of the processes depends upon the work situation. If information is constrained e.g. by unpleasant or risky observational conditions, the process is characterized by a careful, rational consideration of available information in relation to the anatomy and functioning of the system. In the normal work shop conditions, however, the processes are typically based upon general search routines which enable the technicians to map the performance of the system by a rapid stream of judgments of the observations individually. This search is controlled by a representation - mental model - of the system in the general form of a topographic map of typical signal flow routes in electronic circuitry supplying convenient measuring points and references values. 
In this case it has been possible from the verbal protocols to identify basically different processes for the same external task. It illustrates how the process can be controlled by inference rules and models related to the anatomy and function of the specific system as well as by algorithms specifying the process itself using only rudimentary models of the system.

The replacement of complex reasoning by general algorithms relieve the cognitive strain, and a pronounced fixation of the familiar routines was found. Although the mental processes were consciously monitored and verbalized, they appear not to be controlled by conscious decisions, but rather by subconscious conditioning, chaining familiar routines according to the law of least resistance. Familiar search routines were used also in cases for which careful consideration of the information available could lead to efficient short cuts.

Comparing the results of analysis of protocols from control rooms and from maintenance shops, the conclusion is that the behavior of skilled personnel only in special task conditions can be modeled by a sequential algorithmic process description derived directly from verbal protocols, as it has been done for subjects in games and problem solving tasks (Newell and Simon, 1972). In most circumstances the analysis of verbal protocols collected from process operators can only serve as a guide or intermittent indication of his activity at several levels.

\section{MAPPING MENTAL ACTIVITY}

The efficiency of skilled performance is due to the ability to compose the process needed for a specific task as a sequence of familiar subroutines which are useful in different contexts. This implies the existence of links in the sequence at standard key nodes or "states of knowledge" which are characteristic of the specific skill. The data process stops at such links, the mode of processing and frequently the level of abstraction changes, and to study and identify the processes, the activity must be structured according to such key nodes.

First of all, the activity should be broken down to "sub tasks". Sub tasks can be defined at many levels due to the hierarchical structure of the operator's total task. As a preliminary description for the present purpose we have defined a sub task as the sequence of data processing steps and related states of knowledge which connect the initiation of operator response with the resulting manual action, e.g. the response following a warning signal. The number of steps in this sequence depends strongly upon the skill of the operator in the specific task. Therefore, as a general description typically used "states of knowledge" from the protocols are arranged in the sequence which a novice would have to follow. Study of actual, skilled performance may then result in a description in terms of shunting leaps within this basic 
sequence, as illustrated by figure 1 . The diagram resembles a "ladder of abstraction". One leg upwards for analysis of a situation, another downwards for planning of the proper actions. Short cuts from habits and rules connect the two legs of the ladder.

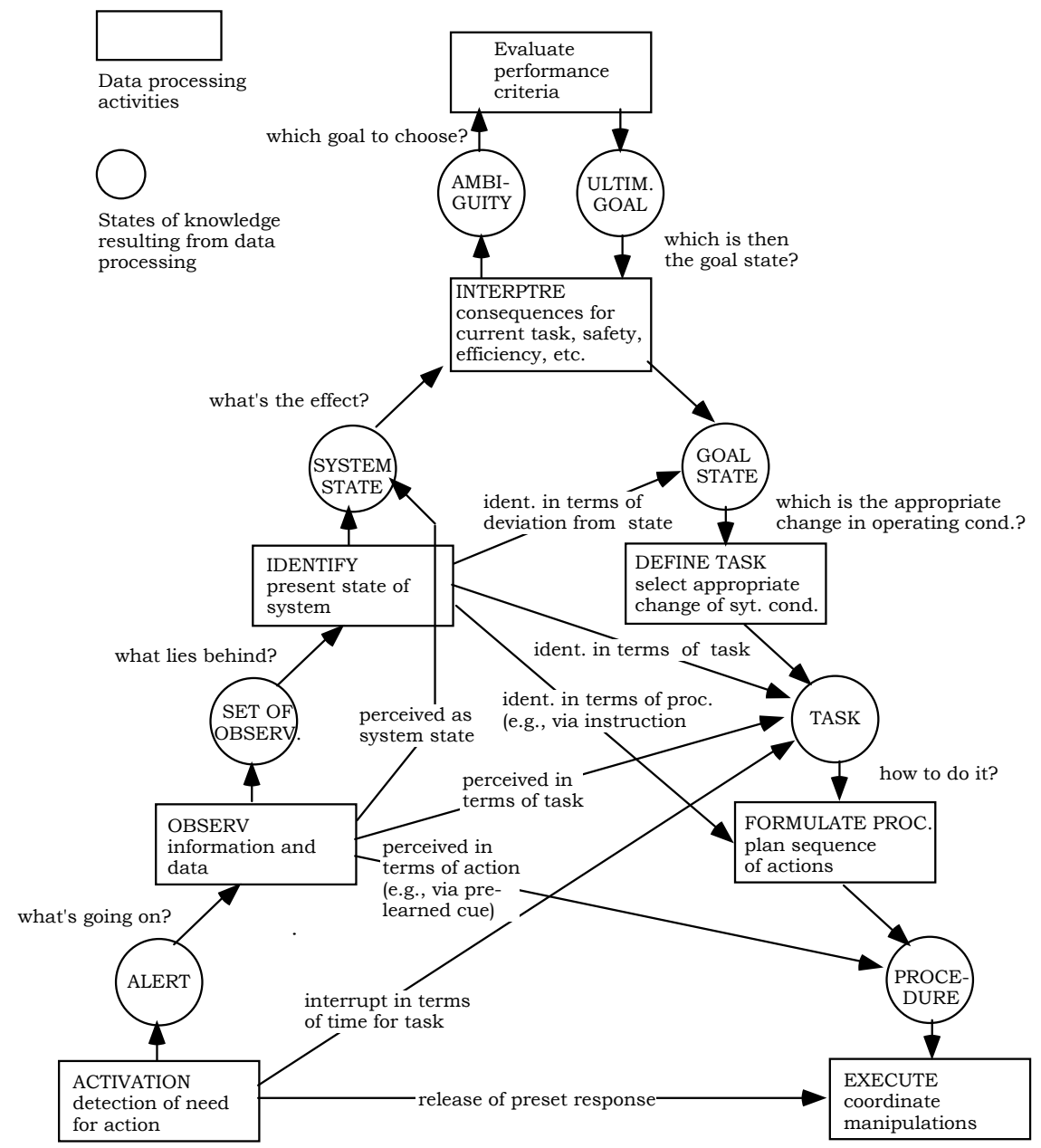

Figure 1. Schematic map of the sequence of mental activities between initiation of the response and the manual actions. The diagram illustrates typical shunting routines in skilled performance. Associative leaps directly between states of knowledge are not shown.

Frequently, a skilled operator does not enter the sequence at its entry; his process feel can initiate consideration first of a step later in the sequence, he may change the order of the steps, and he only occasionally has to move through all the steps in the basic sequence. Two types of shunting effects appear to be active. One is mental activity at a symbolic level leading directly to state of knowledge later in the sequence, e.g. holistic perception leads to observation directly in terms of system state or task to perform rather than observation of separate items of information. Another shunting mechanism appears to be associations based upon experience leading to leaps directly from one state of knowledge to another. 
Detailed expression of sequential, conscious data processing is only found if uncertainty or ambiguity is recognized by the operator. If not, either a leap in the sequence takes place or a state is accepted and the sequence stopped.

The leaps are equivalent to a shunting-out of activities at higher levels of abstraction which call for complex conscious reasoning; as a result they give rise to a considerable increase of data handling capacity.

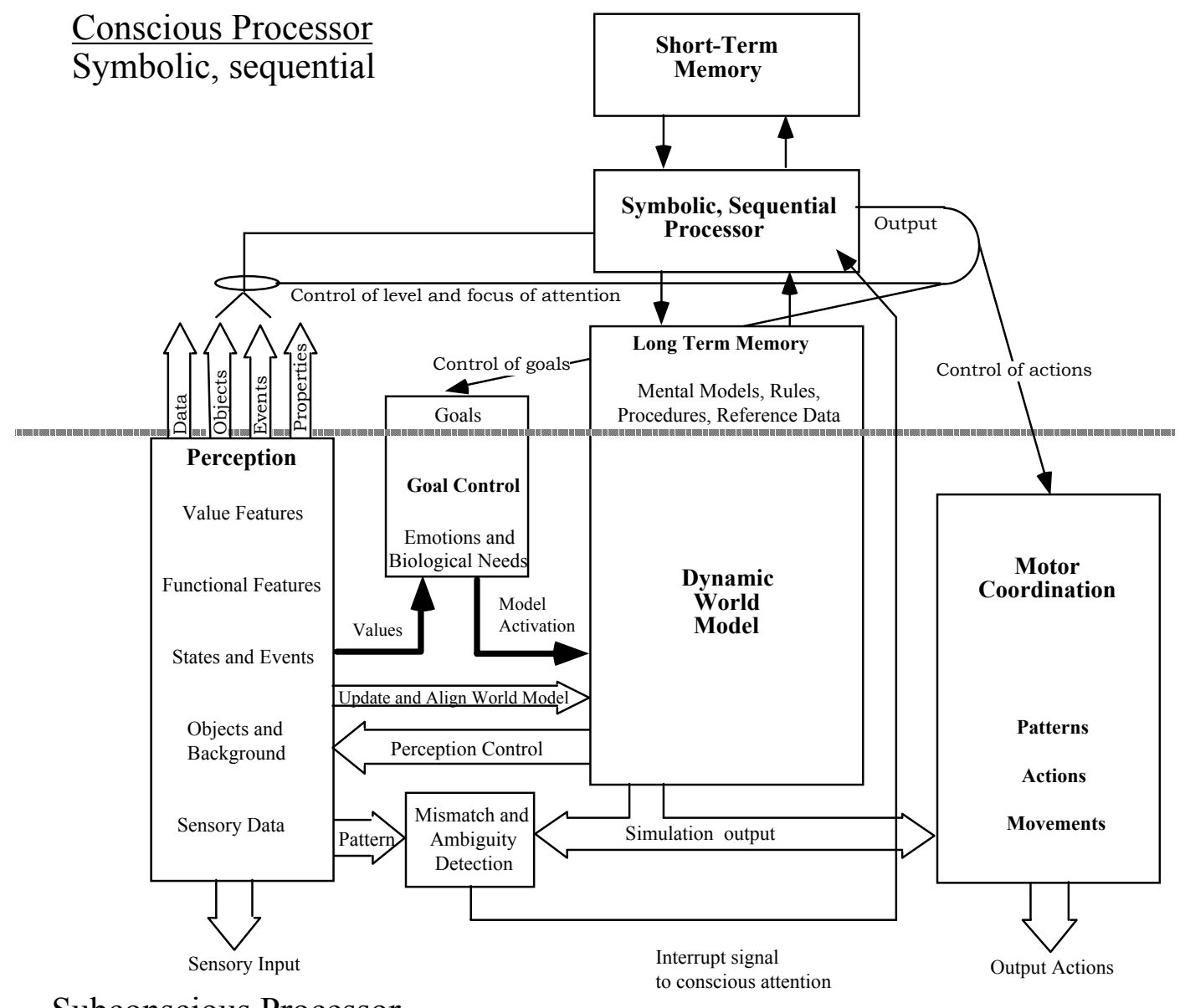

Subconscious Processor

Analog, parallel

Figure 2. Schematic map of the human data-processing functions.

\section{Mapping Human Data Processing Mechanisms}

From the proceeding discussion it follows that a variety of human data processing mechanisms is used for the mental sub task to transform one state of knowledge to the next. A model of these mechanisms and their mutual interaction is needed to support the analysis and description of operator behavior. The model must take into ac count a wide range of mechanisms including rational problem solving as well as intuitive and associative reasoning and sensory-motor skills. A tentative map to serve this purpose is shown in figure 2. The diagram outlines a hybrid model comprising two basically 
different data processing systems to represent the low data capacity found in consciously controlled operations, as well as the ability to respond successfully to situations demanding rapid, high-capacity in formation processing (Miller, 1956).

A high-capacity, parallel processing system serves the subconscious processes related to perception, sensory-motor responses etc. It resembles in its performance a goal oriented, self organizing associative network operating by dynamic matching of input information patterns to stored patterns. Value properties of the input patterns - or their verbal representations - controls the process through a global conditioning, which activates generic stored patterns. These patterns constitute an analog representation of the behavior of the environment, structured in a time-space domain. This internal world model representing the behavior of the environment and the body ac counts for the ability of efficient feed-forward control of the body in sequences too rapid to allow for sensory feedback - "the serial order in behavior" (Lashley, 19 1), for the "process-feel" of the operator, and it directs and controls his attention. If a mis-match occurs, i.e. if the behavior of the environment deviates from the predictions of the model or a relevant model i5 not available, this is detected and the conscious processor is alerted.

The conscious processor is an extremely versatile sequential processor of limited speed and capacity. It acts as a high-level coordinator of the subconscious processes and functions in unfamiliar situations demanding unique responses. The variables treated by the conscious processes can be fetched from the perceptive system at different levels of abstraction, or be purely symbolic variables. The processes can be based upon different types of representations - mental models - of the physical system considered depending upon the task, e.g. representation of system anatomy and function as causal models used for rational deductions; representation of typical system behavior as used in "visual thinking"; or processes controlled by prescribed algorithms - cook book recipes - rather than a structured model.

The two processing systems cooperate, and the subconscious processor by its large repertoire of automated subroutines relieves the limited conscious capacity. The conscious data process controls a sequence of such subroutines by an executive program in which the level of abstraction rises as training increases the complexity and efficiency of the subroutines. This control, however, is significantly influenced by the subconscious processes directing the attention, supplying intuitive hypotheses etc. The system engineering implications of the model suggested and its psychological references are discussed in more detail elsewhere (Rasmussen, 1974).

In common life tasks evolution has fitted man to an efficient interaction with a time-space environment, for which high capacity parallel processes take care of the lower functions of the ladder of abstraction. Abundance of 
redundant information supports feature extraction and the formation and synchronization of the internal world model.

In process control, however, an operator controls a physical process from which only pre-selected information is presented to him and typically as symbolic representation of variables individually. Designers often suppose that he uses rational, conscious processes also at the lower level tasks, which would lead to complex time sharing between tasks at different process levels. An important task when studying process operator performance is to identify the repertoire of ingenious tricks which evolve in operators to avoid this: he adopts focusing strategies (Bruner et.al. 19 6) based upon a model of normal state, leading to judgments of individual observations separately; he synchronizes his world model by convenient information which may be secondary, as relay clicks etc.; he relies on intuitive judgments which, due to the nature of the subconscious world model may be based upon representativeness rather than being rationally founded (Tversky and Kahneman, 1974); etc.

Fixation to such normally very efficient routines when the task condition changes during abnormal plant operation is bound to lead to difficulties, and operator's response to plant malfunction can only be studied in the light of their normal skilled performance. We have to consider processes and mechanisms of basically different nature which are normally studied separately, whereas the problem in our context is largely related to their interaction.

\section{Data, Models, AND PRocedures}

A study of this interaction must be based upon a description of the different types of processes according to a common set of concepts related to the processes, rather than to psychological or neurological descriptions. For this purpose the data processes used to control human interaction with a physical system can be characterized within the following aspects: data, representing the state of variables in the systems; a model, representing the structure of the system constraining the interrelationship of data; and procedures or rules to control the data process.

So far, we have identified the following types of models to be considered in our analysis:

-"Physical Models", representations identifying potential data sources and mapping their spatial relations, e.g., scale models, topographic maps, anatomical diagrams. Frequently used sets of sources can be aggregated into objects at several levels, e.g. components, units, sub-systems. Such models are typically used when the process is controlled by a specific, detailed process algorithm. (Such models are used by Luria's mnemonist to structure the memory (Luria, 1959)). 
-"Functional models" identify potential sources of information together with rules or laws specifying the relationship between in formation from the sources. Such a model can be structured according to the system by aggregating sources into objects such as boilers, pumps, etc. or it can be structured by aggregating sources into functions according to general relations such as mass-balance, feed-back loop etc. The relational rules can be related to the system, being general causal laws, or to the data process used, being e.g. calculating algorithms or heuristic rules. This type of model is found in sequences related to prediction of system behavior or testing of hypotheses.

-"State models" identify sources of information together with consistent sets - snap-shots - of variables representing typical system states, e.g. "normal operation", "power failure". State models are used when preparing a system for a planned operation, during fault location, etc.

-"Behavioral models" are like dynamic, animate state models and are formed by storing system behavior from typical response situations, structured in familiar objects and their generic behavior. The subconscious world model controlling e.g. sensory -motor responses and animate imagery is placed in this category.

The variables describing the state of a physical system can be represented by data in the mental processes in various ways and at several levels of aggregation or abstraction.

Continuous, quantitative - analog - representation of individual variables in the subconscious modeling system seems to be necessary to account for the precision of sensory-motor responses and furthermore seems to be used in "visual thinking". Discrete, quantitative - digitized - representation of individual variables can be used in symbol manipulating algorithms. Typically, however, qualitative representations are used in verbal sequences relating variables to a reference state model, e.g. "high", "abnormal", "hot", "rising".

In higher level verbal sequences collective representation of sets of variables are normally used. They are expressed as verbal labels representing system states or functions in relation to reference state models, such as "air system normal", "boiler ready for start up". Verbal statements at this level very likely are also used to initialize and activate process models, e.g. for visual thinking (Bugelsky, 1970) or for judgment of observations.

The need for procedural rules to control a specific data process depends upon the nature of the model available. If the process is based upon a complex active model performing parallel processing no algorithm is needed to control a specific process; the process is completely specified by the elements and the structure of the model - the problem is transferred to an analog, physical system and the process control left to nature. The subconscious data processes have these features. The neural network is capable of the active modeling needed, considered as a distributed, active net work op- 
erating by excitation interference pattern (Lashley, 1942) and some kind of "holographic" memory (Pribram, 1966). The models can be initiated by a global conditioning of the network through excitation by a reference system which is activated by sensory in formation patterns or by "verbal labels".

The sequential conscious data processes must be based upon stationary models and procedural rules are therefore necessary to specify and control the individual processes. If the model is a detailed functional model the procedures can be very general. If, however, the model is more rudimentary, such as state models or physical models, the procedures will be more specific and implicitly represent system properties. The low data capacity of sequential processes is compensated by the extreme flexibility which includes also the ability of operations on models, e.g. transformation to other models for which procedures or solutions are known.

\section{Conclusion}

Analysis of data processes performed by trained process operators must be based upon identification of the structure of the underlying memory for system properties which includes dynamic pat terns of behavior, structured sets of data and causal relations, as well as rules for actions and operations; categories which are compatible with memory structures identified by psychological experiments (Piaget and Inhelder, 1973). From this and the "reallife" conditions of the study it follows that the appropriate method of analysis depend strongly upon features of the operator's specific task conditions. Methods developed within different professions have to be considered, ranging from linguistics and cognitive psychology to information and computer science and control theory.

When entering a study of human performance in real-life tasks one rapidly finds oneself "rushing in where angels fear to tread". It turns out to be a truly interdisciplinary study for which an accepted frame of reference has not yet been established, and iteration between rather general hypotheses, test of methods, and de tailed analysis is necessary. The work presented here illustrates our approach to the first steps in this process. From analysis of a number of protocols the outlines of a frame of reference compatible with our systems engineering concepts is suggested. Currently we are planning the next phase which involves collection and detailed analysis of protocols and interviews from selected task sequences and simulator experiments to verify models of more specific aspects of operator behavior.

\section{REFERENCES}

Bruner, J. S. Goodnow, J. J. and Austin, G. A. (1956), A Study of Thinking. Wiley, New York.

Bugelsky, B. R. (1970), Words and Things and Images. Am. Psychol. 7, 25, 1002-1012. 
Cornell, C. E. (1968), Minimizing Human Errors. Space Aeronautics, 49, March, 72-81.

Lashley, K. S. (1942), The Problem of Cerebral Organization in Vision. In: Visual Mechanisms, H. Kluver (ed.), Cattell, Lancaster.

Lashley, K. S. (1951), The Problem of Serial Order in Behaviour. In: Cerebral Mechanisms in Behaviour, L. A. Jeffress (ed.). Wiley, New York.

Luria, A. R. (1969), The Mind of a Mnemonist. Jonathan Cape, London.

Miller, G. A. (1956), The Magical Number Seven Plus or Minus Two. Psychol. Rev. 63, 8197.

Newell, A. and Simon, H. A. (1972), Human Problem Solving. Prentice Hall, New Jersey.

Piaget, J. and Inhelder, B. (1973), Memory and Intelligence. Routledge and Kegan Paul, London.

Pribram, K. H. (1966), Some Dimensions of Remembering. In: Macromolecules and Behaviour, J. Gaito (ed.). Appleton, New York.

Rasmussen J. (1959), Man-Machine Communication in the Light of Accident Records. Int. Symp. on M-M Systems, Cambridge. IEE Conf. Records No. 69 (58-MMS. Vol 3).

Rasmussen, J. and Jensen, A. (1974), Mental Procedures in Real Life Tasks: A Case Study of Electronic Trouble Shooting. Ergonomics, 17, No. 3, 293-307.

Rasmussen, J. (1974), The Human Data Processor as a System Component. Bits and Pieces of a Model, Risø-M-172?.

Scott, R. L. (1971), A Review of Safety Related Occurrences in Nuclear Reactors from 19671970. ORNL-TM-3435.

Tversky, A. and Kahneman, D. (1974), Judgment under Uncertainty: Heuristics and Biases. Science, 185, 1124-1131.

\section{Discussion}

\section{Moray:}

What part of the information processing mechanism is accessible by protocol analysis?

\section{Rasmussen:}

So far I don't know . A simple analysis of the verbal sequence in protocols from skilled operators will only give the structure of a higher level strategy controlling a sequence of - possibly subconscious - subroutines. But a more detailed analysis of the intuitive guesses used by the operators and a linguistic analysis of their statements can possibly give information on the structure and elements of the "world model" behind their subconscious subroutines.

\section{Drury:}

Do you know of any studies showing that verbal protocols do not interfere with the process control task?

\section{Rasmussen}

No - and this interference was one of the problems we expected to meet. However, in a study of the mental procedures used by electronic troubleshooters we discussed the procedures and possible improvements with the 
technicians after the analysis of some 30 protocols without finding any significant change of the procedures used in the following protocols .

My conclusion is, that it is rather difficult to interfere with the procedures of a skilled person in his normal task - if the verbalization interferes, he rather stops talking, which is also a problem. 